\title{
Mapas Conceptuales como Herramienta en la Enseñanza de Contenidos Comunes a Diferentes Cursos de Ingeniería Agronómica
}

\author{
Miriam E. Presutti, Elisa C. Miceli y Gladys M. Laporte \\ Universidad Nacional de La Plata, Facultad de Ciencias Agrarias y Forestales, Diagonal 113 esq \\ 61 No 469 Piso 2, (1900) La Plata, Buenos Aires-Argentina (e-mail: presutti@agro.unlp.edu.ar)
}

\begin{abstract}
Resumen
En este trabajo se muestran las ventajas de la utilización de mapas conceptuales en diferentes cursos de la carrera de Ingeniería Agronómica al abordar un mismo contenido. La temática elegida para su aplicación es la Fusariosis de la espiga de trigo (FET), enfermedad producida por un hongo que afecta al cultivo y cuya aparición se produce ante determinadas condiciones ambientales. El mapa conceptual de la FET fue elaborado usando los conceptos extraídos de los textos utilizados en los diferentes cursos y usando el programa CmapTools. El mapa finalmente fue exportado a formato de página Web, para que todos los estudiantes accedan fácilmente a él desde su propia computadora. Se concluye que al utilizar el mapa conceptual al inicio de la clase en cada curso donde se trate el tema, el alumno adquiere una visión general del mismo, continuando la clase con la profundización de los temas específicos de cada área.
\end{abstract}

Palabras Claves: mapa conceptual, CmapTools, fusariosis, trigo, ingeniería agronómica

\section{Concept Maps as a Teaching Tool of Similar Subjects in Agronomy Engineering Courses}

\begin{abstract}
In this work the advantages of using concept maps as a tool in covering the same subjects in different courses of Agronomy Engineering are shown. The selected topic to construct the concept map is the Fusarium ear blight (FEB), a fungal disease that affects ear wheat and it appears with particular environmental conditions. The concept map of FEB was developed using the concepts extracted from the textbooks used in different courses and applying the software CmapTools. Finally, the map was exported to Web page format so all the students could access it from their own computer. It is concluded that using the concept map at the beginning of the class in each course where the selected concept is treated, students can get a global vision of it, to then deal with the specific subjects of the course in depth.
\end{abstract}

Keywords: concept map, CmapTools, fusariosis, wheat, agronomy engineering 


\section{INTRODUCCIÓN}

El mapa conceptual es una técnica cada día más utilizada en diferentes niveles de la educación (Nesbit y Adescope, 2006). Se lo utiliza como herramienta para el aprendizaje y la evaluación, permitiéndole al docente explorar los conocimientos previos de los alumnos; mientras que, al alumno le permite organizar, interrelacionar y fijar el conocimiento del contenido estudiado. Se lo ha utilizado en la evaluación a nivel de cursos y de programas (Turns et al., 2000). También para transmitir y compartir experiencias personales en investigaciones que requieren datos cualitativos (Wheeldon y Faubert, 2009).

Los mapas conceptuales, desarrollados por Novak y Gowin (1984), se usan como un medio para la descripción y comunicación de conceptos dentro de la "Teoría de Asimilación" (Ausubel et al., 1978). La teoría está basada en un modelo constructivista de los procesos cognitivos humanos. El aprendizaje significativo pone énfasis en la creación, evolución y relación entre los conceptos. Aunque Ausubel no menciona a los mapas conceptuales en su teoría, los mismos fueron desarrollados para promover el aprendizaje significativo. Un aprendizaje es significativo cuando una nueva información (concepto, idea, proposición) adquiere significados para el estudiante a través de una interacción con la estructura cognitiva preexistente. La interacción entre el nuevo conocimiento y el ya existente, produce la modificación de ambos. El aprendizaje es un proceso dinámico y aprender significativamente implica atribuir significados con componentes personales. El aprendizaje sin atribución de significados personales, sin relación con el conocimiento preexistente, es memorístico, no significativo.

Un mapa conceptual es una representación gráfica de un conjunto de conceptos y las relaciones entre ellos sobre un dominio específico del conocimiento. En este esquema, los conceptos se representan como nodos rotulados y las relaciones como arcos rotulados. Novak y Gowin (1984) indican que los mapas conceptuales "tienen por objeto representar relaciones significativas entre conceptos en forma de proposiciones". Estos autores, indican que los conceptos más generales o inclusivos deben representarse en la parte superior del mapa, y los más específicos o menos inclusivos, en la inferior. La jerarquía en la disposición de los conceptos, minimiza la desorientación en el procesamiento de los mapas conceptuales, independientemente del conocimiento previo que posean los alumnos sobre el tema (Amadieu et al., 2009). La creación de conocimiento requiere un nivel alto de aprendizaje significativo, y los mapas conceptuales facilitan el proceso de creación de conocimiento en los estudiantes en una disciplina. Los educadores han reconocido que el proceso de crear un mapa conceptual es lo importante, no solamente el resultado final (Novak, 1990; Novak y Cañas, 2006).

La construcción de un mapa conceptual se inicia con una pregunta central la cual se responderá con el contenido del mapa. Generalmente las preguntas que requieren una explicación, en vez de una simple descripción, Ilevan a construir mejores mapas conceptuales (Novak y Cañas, 2007). Son preferibles aquellas preguntas que requieran un pensamiento profundo y significativo que las que solamente describen objetos (Derbentseva et al., 2007).

Novak (1995), describe una amplia variedad de aplicaciones de los mapas conceptuales en la enseñanza. Existen diversos proyectos en los que se utilizan los mapas conceptuales ideales, aquellos elaborados por expertos y científicos, generalmente almacenados en servidores de contenido público conectados a la red Internet y accesible mediante la Web. Como ejemplos, se pueden citar el Sistema de Conocimiento de Ciencias (Astronomía, Física, Química y Biología) desarrollado por Cañas et al. (2003). El mismo consta de un conjunto de mapas conceptuales, donde se relacionan a las unidades temáticas de los mapas con recursos hipertextuales y multimediales. Otro ejemplo es un mapa conceptual sobre la exploración de Marte, que fuera desarrollado por expertos de la NASA (Briggs et al., 2004) que consiste en una colección de más de 100 mapas conceptuales sobre dicho planeta. Chen et al. (2008), proponen la construcción de un mapa conceptual utilizando como nodos a las palabras claves de artículos y conferencias sobre un tema determinado y también medir la fuerza de las relaciones entre conceptos, de modo de guiar a los estudiantes hacia los temas que posean mayor relación con el tema en estudio. 
La experiencia muestra que la diferencia en la representación del conocimiento entre expertos y estudiantes es significativa (Williams, 1998; Markham y Mintzes, 1994). En los mapas conceptuales ideales creados por expertos y científicos, la organización del contenido está basada en la sabiduría que los ha llevado a un nivel de entendimiento de su área de investigación, mostrando relaciones con otras áreas que no son tenidas en cuenta por otras personas. Estos mapas conceptuales ideales pueden usarse como herramienta de enseñanza y ayudar al estudiante en el aprendizaje de contenidos (Hilbert y Renkl, 2009; Chularut y DeBacker, 2004). Aunque la construcción de éstos mapas, al requerir el consenso entre los diferentes expertos, insume mayor tiempo (Hsu y Hsieh, 2005).

Mediante la utilización de recursos tecnológicos digitales es posible incrementar el poder de los mapas conceptuales al agregar otros recursos asociados a los conceptos, como imágenes, documentos, páginas Web o fórmulas De éste modo el alumno puede ir recorriendo la escala Abstracción/Iconicidad. La escala de iconicidad más conocida es la que Abraham Moles plantea dividida en doce grados, que van desde la máxima iconicidad (el propio objeto que se designa) hasta aquellas imágenes de iconicidad nula (descripción del objeto mediante palabras o fórmulas algebraicas). El principio que rige esta escala decreciente radica en que el "grado de iconicidad" de una forma es inverso a su "grado de abstracción", comparando siempre la imagen con el objeto (Moles, 1991).

En el diseño curricular de la carrera de Ingeniería Agronómica, una situación problemática identificada está relacionada con aquellos contenidos transversales que son tratados en distintas asignaturas desde diferentes perspectivas. En esos casos el estudiante va adquiriendo conocimientos parciales en cada uno de los cursos y al no disponer de una visión general del tema, no realiza la debida interrelación de los contenidos. En este marco, la temática elegida para realizar un mapa conceptual es la Fusariosis de la espiga de trigo (FET), contenido abordado en varias asignaturas.

El objetivo general del presente trabajo es elaborar un mapa conceptual digital de la FET a partir de los textos utilizados en el dictado de los distintos cursos de la carrera donde se aborde ésta temática desde diferentes áreas del conocimiento y exponer las ventajas de su utilización en un entorno de aprendizaje por hipertexto. Los objetivos específicos son: i) Buscar asociaciones entre los diferentes conceptos con el fin de mejorar los procesos de aprendizaje de la temática; y ii) Crear y asociar al mapa otros recursos: páginas Web, imágenes, esquemas, textos y simulaciones, que mejoren el proceso de la enseñanza.

\section{METODOLOGÍA}

Una vez identificada la pregunta a responder con un mapa conceptual, en este caso la FET, las etapas metodológicas seguidas para su construcción fueron:

i) Listado de los conceptos: se realiza un listado de los conceptos (palabras, hechos o ideas) relacionadas con el tema) surgidos a partir de la lectura de los textos y de las experiencias personales. En ésta etapa no se tiene en consideración la redundancia de conceptos, la importancia relativa o las relaciones entre ellos.

ii) Organización: Se reúnen los conceptos en grupos y subgrupos, identificando términos que representen jerarquías. Se incluyen en ésta etapa posibles términos inicialmente omitidos.

iii) Diseño: Se acomodan jerárquicamente los conceptos de modo de representar las interrelaciones entre los grupos y subgrupos.

iv) Conexión: Utilizando líneas y flechas se unen conceptos. Se escribe una palabra o frase corta sobre cada relación. Las proposiciones resultantes contienen dos o más conceptos conectados que forman un enunciado con sentido.

v) Finalización del Mapa Conceptual: Controlando que todos los conceptos y las relaciones sean correctas y que no falten conceptos importantes. Se ubican las imágenes, hipertextos, ecuaciones que complementen o le dan mayor significado a los conceptos o proposiciones. 
Un mapa conceptual, se construye de forma iterativa, una vez construido es necesario revisarlo y agregar nuevos conceptos y relaciones. Se arriba a un resultado final luego de tres o más revisiones, por lo cual utilizar un programa de computadora diseñado específicamente para ello facilita la tarea. El mapa conceptual fue elaborado utilizando el programa CmapTools desarrollado por el Instituto de Investigaciones Cognitivas en Humanos y Computadoras (Institute for Human and Machine Cognition, IHMC), de la Universidad de West Florida (Estados Unidos). El programa CmapTools (Cañas et al., 2004), es una herramienta de descarga gratuita. El programa permite agregarle recursos (fotos, imágenes, gráficos, videos, esquemas, tablas, textos, páginas Web u otros mapas conceptuales) para explicar más profundamente sus contenidos.

\section{DOMINIO DE CONOCIMIENTO Y MAPA CONCEPTUAL}

La fusariosis de la espiga de trigo (FET) es una enfermedad producida por un hongo (Fusarium $s p$.) que afecta el cultivo del trigo, específicamente durante el momento de floración; su aparición se produce ante determinadas condiciones ambientales (temperatura, humedad y precipitación). Este hongo produce micotoxinas que permanecen en el grano y su presencia afecta la salud humana y animal (aspecto tratado en el curso Agroindustrias), los métodos de control de la enfermedad en el cultivo son abordados en el curso de Terapéutica vegetal mientras que con los elementos tratados en el curso de Geoinformación se aplican modelos de pronóstico de la enfermedad basado en condiciones ambientales. Además, se incluyen en el mapa conceptual los conocimientos de otra disciplina, la Fitopatología, donde se abordan todos los aspectos referidos al agente causante de la enfermedad.

La FET es una enfermedad difundida mundialmente en áreas productoras de trigo, cuando coinciden los estados de espigazón, floración e inicio de llenado del grano con períodos muy húmedos y templados-cálidos, y además el patógeno que la ocasiona se encuentra presente en el lote. Las pérdidas ocasionadas al cultivo de trigo están relacionadas con la esterilidad de las espiguillas y el desarrollo anormal de granos, que son castigados en la comercialización pues tienen una constitución alterada y bajo peso hectolítrico. Además de estas pérdidas cuantificables en el rendimiento de trigo, en los granos infectados se pueden formar micotoxinas (metabolitos producidos por el hongo) que afectan tanto a la salud humana como a la animal. Se detallan a continuación los cuatro (4) grupos de conceptos inicialmente reconocidos para la construcción del mapa conceptual: Presencia del patógeno, Estado de desarrollo del cultivo, Condiciones ambientales predisponentes y Control de la enfermedad. Dentro de cada grupo se irán incorporando otros conceptos.

\section{Presencia del patógeno}

La fusariosis de la espiga es una enfermedad causada predominantemente por el hongo Fusarium graminearum cuya forma sexual es Gibberella zeae. El patógeno sobrevive en el campo en restos de cultivos anteriores y malezas formando estructuras denominadas peritecios. La velocidad de descomposición de los restos del cultivo es importante para la supervivencia del hongo. En el interior de los peritecios, que tienen una coloración púrpura a negra se forman las ascosporas. Las mismas son transportadas por el viento hasta alcanzar las espigas de los cereales, depositándose en las anteras iniciándose de éste modo el proceso de infección. Algunas prácticas agronómicas, como la no rotación de cultivos o sistemas de labranza conservacionista pueden favorecer las condiciones para que el patógeno sobreviva en el lote, provocando nuevas infecciones.

\section{Estado de desarrollo del cultivo}

Una vez que se inicia la infección del hongo en las flores, la enfermedad se evidencia en las espigas. Los síntomas son una decoloración prematura de las espiguillas infectadas, pudiendo llegar a tomar toda la espiga. Las infecciones que ocurren más tempranamente generalmente causan el aborto de las flores y por lo tanto el grano no se desarrolla; mientras que aquellas infectadas más tardíamente desarrollarán granos anormales (arrugados o chuzos). Esto disminuye el rendimiento del cultivo, pues hay menor número de granos por espiga y además los granos son de menor tamaño. Esto lleva a que el productor reciba menos dinero en la comercialización de su cosecha. Si la infección ocurre luego del llenado del grano, aunque el desarrollo del mismo no es 
afectado, el hongo está presente y los niveles de la micotoxina pueden llegar a ser significativos, estos granos contaminados son perjudiciales a la salud si son consumidos como alimentos (Peraica et al., 2000).

La calidad industrial también se ve afectada porque el hongo es capaz de destruir los gránulos de almidón, las paredes celulares y las proteínas del endosperma. Todo ello redunda en una menor capacidad de molienda para la elaboración de panificados y pastas. Sin embargo, la característica más relevante de este hongo es la capacidad de producir micotoxinas, denominadas DON (deoxinivalenol) y ZEA (zearalenona). Las toxinas de los hongos se diferencian de las de origen bacteriano, dado que éstas últimas, en su mayoría son macromoléculas (proteínas, polisacáridos, etc.) mientras que las micotoxinas son compuestos de peso molecular bajo. Por otra parte su química puede ser compleja y presentan una estabilidad frente a agentes físicos y químicos que las hacen muy difíciles de eliminar una vez que han sido producidas en los alimentos.

La presencia de hongos no implica necesariamente la presencia de micotoxinas, pero sí una posible contaminación. Para que los alimentos con DON representen un riesgo a la salud humana deben ser ingeridos en grandes cantidades. Si bien el DON no es muy tóxico, tampoco es inocuo ya que inhibe la síntesis de proteínas. Se debe tener en cuenta que el DON no se distribuye homogéneamente en el grano, su concentración es mayor en la "cáscara" por lo que alimentos con salvado constituyen un mayor riesgo a la salud. La otra micotoxina producida por el Fusarium es la ZEA, tiene efectos estrogénicos y anabólicos. En vacas lecheras expuestas a ZEA se ha observado una disminución del porcentaje de preñez y, al parecer, el paso de metabolitos a la leche es mínimo.

\section{Condiciones ambientales predisponentes}

El desarrollo de esta enfermedad depende fundamentalmente de las condiciones ambientales imperantes durante el momento de la floración del cultivo, tales como temperaturas entre 20 y 30 ${ }^{\circ} \mathrm{C}$, lluvias y alta humedad relativa con períodos de mojado de las espigas de 48 a 60 horas. La ocurrencia de la enfermedad en la región Pampeana, ha impulsado el desarrollo de modelos de pronóstico de la enfermedad, con base meteorológica. Estos modelos buscan apoyar la definición de estrategias de manejo y toma de decisión para el control químico, mediante la aplicación de fungicidas (Moschini et al., 2002).

En la región pampeana opera el sistema de pronóstico del grado de riesgo de FET a través de los registros diarios de temperatura máxima y mínima $\left({ }^{\circ} \mathrm{C}\right.$ ), humedad relativa (promedio porcentual de las observaciones correspondientes a las 8,14 y $20 \mathrm{~h}$ ) y precipitación (mm). En base a estos datos se han construido modelos predictivos que se corren tres días por semana, estimando el nivel de enfermedad. Este grado de riesgo se categoriza en alto, moderado y bajo, y se confeccionan mapas respectivos que son ofrecidos en sitios Web de organismos nacionales. El riesgo alto indica que es aconsejable realizar un control inmediato con fungicida (en no más de uno a dos días). El riesgo moderado alerta sobre la ocurrencia de eventos meteorológicos recientes que no conducirían a inmediatas aplicaciones químicas. El riesgo bajo implica que los modelos presentan muy bajos a nulos valores predichos de incidencia de la Fusariosis. A pesar de que la Fusariosis es una enfermedad monocíclica cuyo inóculo inicial lo constituyen las ascosporas del hongo provenientes de los restos culturales, su severidad final resulta de infecciones múltiples originadas en episodios de inoculación ocurridos durante el período crítico del trigo, complicando su control químico. Este sistema de pronóstico permite cuantificar la evolución del proceso múltiple de infección (evento por evento).

\section{Control de la enfermedad}

Hasta el momento, ninguna de las estrategias de control utilizadas para otras enfermedades han mostrado suficiente eficacia sobre la fusariosis. La resistencia genética es escasa y difícil de incorporar en variedades de alta productividad y calidad. (Galich, 2002) Las labranzas conservacionistas, especialmente la siembra directa, ocupan una importante y creciente superficie en la región pampeana norte y los beneficios agronómicos indudables de esta modalidad de producción fuerzan a buscar soluciones que limiten la supervivencia del patógeno y su dispersión. 
La alternativa del control químico presenta dificultades en su implementación y no siempre justifica la inversión. Finalmente, esta enfermedad es altamente dependiente de las condiciones climáticas y sobre esta realidad, el hombre no tiene influencia. Sin embargo, los sistemas de pronósticos a corto y mediano plazo, así como la aplicación del modelo predictivo de incidencia pueden ayudar a tomar decisiones. La única manera de reducir el impacto de la fusariosis en los rendimientos y calidad a niveles tolerables es la integración de técnicas que contribuyan en conjunto a disminuir el nivel de la enfermedad.

\section{Comportamiento varietal}

En el trigo existe variabilidad genética para resistencia a fusariosis aunque no se conoce ningún genotipo inmune. Se han identificado buenas fuentes de resistencia, pero aún no ha sido posible transferirlas en germoplasma de alto rendimiento y calidad.

\section{Control o manejo cultural}

Debido al amplio rango de hospedantes, la elevada capacidad saprofítica de Fusarium graminearum y la dispersión del inóculo a grandes distancias, las prácticas agronómicas tendientes a la eliminación o reducción del inóculo primario, tales como rotaciones con cultivos no hospedantes y labranzas con reducida o nula cobertura de rastrojos no resultan muy efectivas para el control de la enfermedad. Sin embargo, mediante una adecuada rotación y sucesión de cultivos se podría reducir cuantitativamente la fuente de inóculo primario dentro del lote, factor de importancia dado el rol preponderante de la misma en la infección en condiciones de epidemia.

\section{Control químico}

El objetivo del tratamiento es la protección de la principal vía de acceso del patógeno a la flor, que son las anteras y debe ser realizado en forma preventiva, es decir antes de que se manifiesten los primeros síntomas. Los fungicidas disponibles en el mercado para control de fusariosis presentan a campo una moderada a baja eficacia de control pues es difícil de alcanzar las anteras con la pulverización debido a la estructura de la espiga. El uso de fungicidas es una medida complementaria que antes de implementarse exige ciertas consideraciones: 1) Diagnóstico de la enfermedad, 2) Umbral de daño económico y 3) Momento, número y formas de aplicación del funguicida.

\section{RESULTADOS Y DISCUSIÓN}

Siguiendo los pasos enumerados anteriormente se obtiene el mapa conceptual de la FET. El mismo está organizado en cuatro (4) grupos de conceptos: Presencia del patógeno, Estado de desarrollo del cultivo, Condiciones ambientales predisponentes y Control de la enfermedad. Cada uno de los grupos presentados corresponde a un área disciplinar distinta y en el mapa de muestran las relaciones entre ellas. Los nodos y las proposiciones presentadas en el mapa conceptual surgen de los textos En la Figura 1 se observa el mapa conceptual construido utilizando el programa CmapTools, y exportado a formato de página Web. El mapa conceptual aquí presentado tiene como finalidad que el estudiante tenga una visión general de las relaciones transversales y verticales de los contenidos curriculares de las diversas asignaturas de un área específica a través de un hilo conductor.

La representación del mapa conceptual es predominantemente visual, permitiendo la percepción global del objeto de estudio, manifiestando la jerarquización y usando expresiones abreviadas y significativas para los conceptos y palabras enlace. En el momento de presentar a un alumno éste mapa conceptual como siendo el mapa correcto estaríamos promoviendo el aprendizaje mecánico en detrimento del aprendizaje significativo. Se pueden observar los íconos inmediatamente debajo de algunos conceptos en el mapa. Cada uno de ellos representa información adicional sobre el correspondiente concepto. Esta información puede estar en la forma de imágenes, texto, páginas Web. La inclusión de hipertexto le permite al alumno navegar libremente a través de todo el contenido y construir activamente su conocimiento. La naturaleza no lineal del mapa conceptual digital provee un alto grado de libertad y acceso rápido a la información que facilita el aprendizaje. 
Al proporcionarle al estudiante un mapa conceptual como pagina inicial en un entorno de aprendizaje por hipertexto se le minimiza la desorientación y la sobrecarga de información lo cual resulta en un mejor aprendizaje.

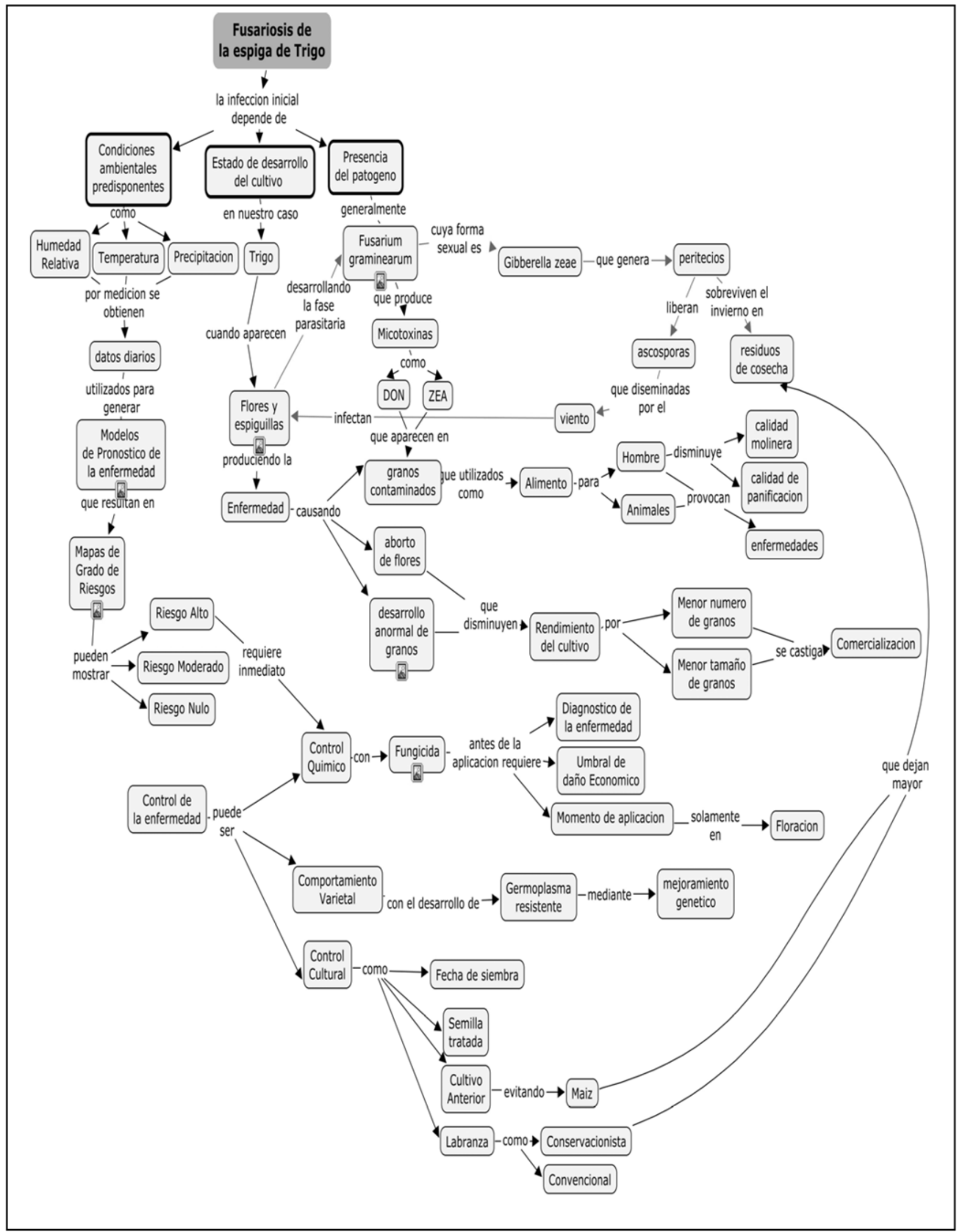

Fig. 1: Mapa conceptual de la Fusariosis de la espiga de trigo realizado con el programa CmapTools. 
Al agregar otros recursos tecnológicos digitales asociados a los conceptos, es posible recorrer la escala Abstracción/Iconicidad, desde el mapa conceptual, situado en la mitad de la escala, puede ver una fórmula (en nuestro caso en el concepto "modelos de pronóstico de la enfermedad"), con lo cual se sitúa en un nivel mayor de abstracción, o bien una imagen (una espiga afectada, la aplicación de un fungicida) con una grado de iconicidad mayor. También puede acceder a documentos que describen en profundidad los conceptos y sus relaciones. Éste mapa en formato digital es entregado a los estudiantes en un CD-ROM para que ellos mismos lo recorran, pudiendo iniciar su recorrido a partir de cualquier concepto. La ventaja es que al tenerlo como página inicial en sus recorridos, la desorientación disminuye, ya que siempre es posible volver al mapa para volver a tener la visión global del tema.

Una de las ventajas de los enfoques interdisciplinares es que ayudan a combatir la tradicional y perjudicial segmentación de las enseñanzas que pueden dar lugar, entre otros inconvenientes, a ideas equivocadas sobre la ciencia y el conocimiento científico como un conjunto de áreas cerradas y autocontenidas y sin interacción recíproca. Es importante reconocer que un mapa conceptual nunca está terminado pues, después que se construye un mapa preliminar, siempre es necesario re-trabajar el mismo, otros conceptos pueden ser agregados, otros mapas, etc. Ésta es una de las razones que demuestra la utilidad de la utilización de programas específicos para la construcción de los mapas conceptuales digitales. Quizá la forma más acertada de utilizar los mapas conceptuales es en el trabajo en grupo y, naturalmente, son los alumnos quienes deben dedicarse activamente a construirlos contando con la mediación del profesor. En este sentido se pueden desarrollar nuevas relaciones conceptuales, en especial si los alumnos tratan activamente de construir relaciones preposicionales entre conceptos que previamente no se consideraban relacionados, ya que cuando se elaboran los mapas se dan cuenta de nuevas relaciones y por consiguiente de nuevos significados, fomentando la creatividad y facilitando la participación.

\section{CONCLUSIONES}

El mapa conceptual construido es utilizado al iniciar la clase donde se aborde este contenido en cada curso, para que el alumno tenga una visión general del mismo. Una vez que se tiene el panorama completo del tema se continúa en la profundización de los saberes específicos de cada área. Los conceptos, al ir relacionándose por medio de las palabras enlace, se van almacenando en la mente de modo organizado y jerárquico de manera que serán más fácilmente comprendidos por el alumno.

Otra ventaja de su implementación como herramienta de la enseñanza de contenidos, es que los alumnos adquieren mayores habilidades durante la lectura de los textos y bibliografías relacionados con cada uno de los temas tratados y extraen los conceptos que resultan esenciales en relación con la tarea propuesta. Si el estudiante cuenta con un mapa conceptual digital como pagina inicial en un entorno de aprendizaje por hipertexto, le permite navegar a través de todo el contenido, minimizándole la desorientación y la sobrecarga de información lo cual resulta en un mejor aprendizaje.

A partir de nuestra experiencia, se recomienda: 1) la incorporación del uso de los mapas conceptuales como estrategias didácticas en las rutinas de las clases, pues fomentan el aprendizaje significativo; 2) Incentivar a los estudiantes en el conocimiento de los mapas conceptuales para facilitarles el aprendizaje; y 3) Difundir el uso de Mapas Conceptuales por parte de los docentes como instrumento de representación de los conocimientos, a modo de síntesis o esquema visual del contenido, mejorando la comprensión, así como el conocimiento estructurado de lecturas, experiencias o cualquier tipo de investigación

Una de las limitaciones de la presente experiencia, se refiere a la dificultad de poder realizar estudios con los estudiantes para confirmar la eficiencia de la estrategia usada, debido a que el tema seleccionado se dicta en asignaturas ubicadas en diferentes años de la curricula de la carrera. Aunque, al incorporar la herramienta dentro de un curso es factible realizar pruebas para medir si los estudiantes son capaces de priorizar información, integrar conceptos y construir nuevos conocimientos. Estas consideraciones serán tenidas en cuenta en futuros trabajos. 


\section{REFERENCIAS}

Amadieu F., T. van Gog, F. Paas, A. Tricot y C. Marine; Effects of prior knowledge and conceptmap structure on disorientation, cognitive load, and learning, Learning and Instruction:19, 376-386 (2009)

Ausubel, D. P., J. D. Novak, y H. Hanesian; Educational Psychology: A Cognitive View (2a edición). New York: Holt, Rinehart \& Winston. Reimpreso, 1986. New York: Warbel \& Peck. (1978)

Briggs G. y otros cinco autores; Concept Maps Applied To Mars Exploration Public Outreach. Concept Maps: Theory, Methodology, Technology, Proc. of the First International Conference on Concept Mapping. Pamplona, España, (2004).

Cañas A.J. y otros seis autores; Entendiendo las Ciencias a Través de Mapas Conceptuales. http://www.virtualeduca.org/2003/es/actas/2/2_08.pdf (2003) Acceso: 28 agosto 2008

Cañas, A. J. y otros cinco autores; CmapTools: A knowledge modeling and sharing environment. In A. J. Cañas, J. D. Novak \& F. M. González (Eds.), Concept maps: Theory, methodology, technology. Proceedings of the first international conference on concept mapping (Vol. I, pp. 125133). Pamplona, España (2004).

Chen N.S. , Kinshuk , C. W. Wei y H.J. Chen; Mining e-Learning domain concept map from academic articles, Computers \& Education: 50, 1009-1021 (2008)

Chularut, P. y T.K. DeBacker; The influence of concept mapping on achievement, self-regulation, and self-efficacy in students of English as a second language, Contemporary Educational Psychology: 29, 248-263 (2004)

Derbentseva N, F. Safayeni y A. Cañas; Concept Map: Experiments on Dynamic Thinking, Journal of Research in Science Teaching: 44 (3), 448-465 (2007)

Hilbert, T.S. y A. Renkl; Learning how to use a computer-based concept-mapping tool: Selfexplaining examples helps, Computers in Human Behavior: 25, 267-274(2009)

Hsu, L. y S. Hsieh; Concept maps as an assessment tool in a nursing course, Journal of Professional Nursing: 21, 141-149 (2005).

Galich, M. T.; Fusariosis de la espiga del trigo: Desarrollo de Cultivares Resistentes, IDIAXXI, (2002)

Markham, K. M. y J. J. Mintzes; The Concept Map as a Research and Evaluation Tool: Further Evidence of Validity, Journal of Research in Science Teaching: 31(1), 91-101 (1994).

Moles, A.; La imagen. Comunicación funcional. Editorial Trillas. México, (1991).

Moschini, R.C, M.T.V. De Galich ; J.G. Annone y O. Polidoro; Enfoque Fundamental-Empírico para Estimar la Evolución del Índice de Fusarium en Trigo, RIA: 31(3), 39-53 (2002)

Nesbit, J.C, y O. Adescope; Learning with concept and knowledge maps: A metaanalysis, Review of Educational Research: 76(3), 413-448 (2006)

Novak, J.D. y D.B. Gowin; Learning How to Learn, Cambridge University Press Cambridge UK, (1984).

Novak, J.D.; The effect of concept mapping to enhance text comprehension and summarization, Journal of Experimental Education: 71, 5 - 23 (1990). 
Novak, J.D.; Concept maps to facilitate teaching and learning, Prospects: 25, 95 - 111 (1995).

Novak, J.D y A. Cañas; Construyendo sobre Nuevas Ideas Constructivistas y la herramienta Cmap Tools para crear un nuevo Modelo para Educación. Institute for Human and Machine Cognition.http://eduteka.org/pdfdir/CmapToolsNuevoModeloEducacion.pdf (2006) Acceso: 30 Agosto 2008

Novak J.D y A.J. Cañas; Theoretical origins of concept maps, how to construct them, and uses in education, Reflecting Education; 3 (1), 29-42 (2007)

Peraica, M., B. Radic, A. Lucic y M. Pavlovic; Efectos tóxicos de las micotoxinas en el ser humano, Boletín de la O.M.S., Recopilación de artículos Nº 2 (2000)

Turns J., C.J. Atman y R. Adams; Concept Maps for Engineering Education: A Cognitively Motivated Tool Supporting Varied Assessment Functions, IEEE Transactions on Education: 43 (2), 164 - $173(2000)$

Williams, C. G.; Using Concept Maps to Assess Conceptual Knowledge of Function, Journal of Research in Mathematical Education, 29(4), 414 - 421 (1998)

Wheeldon J. y J. Faubert; Framing Experience: Concept Maps, Mind Maps, and Data Collection in Qualitative Research, International Journal of Qualitative Method 8(3), 68-83 (2009) 